\title{
The Complexity of Folding Self-Folding Origami
}

\author{
Menachem Stern, Matthew B. Pinson, and Arvind Murugan \\ Department of Physics and the James Franck Institute, University of Chicago, \\ Chicago, Illinois 60637, USA
}

(Received 21 March 2017; revised manuscript received 5 October 2017; published 22 December 2017)

\begin{abstract}
Why is it difficult to refold a previously folded sheet of paper? We show that even crease patterns with only one designed folding motion inevitably contain an exponential number of "distractor" folding branches accessible from a bifurcation at the flat state. Consequently, refolding a sheet requires finding the ground state in a glassy energy landscape with an exponential number of other attractors of higher energy, much like in models of protein folding (Levinthal's paradox) and other NP-hard satisfiability (SAT) problems. As in these problems, we find that refolding a sheet requires actuation at multiple carefully chosen creases. We show that seeding successful folding in this way can be understood in terms of subpatterns that fold when cut out ("folding islands"). Besides providing guidelines for the placement of active hinges in origami applications, our results point to fundamental limits on the programmability of energy landscapes in sheets.
\end{abstract}

DOI: 10.1103/PhysRevX.7.041070

Subject Areas: Metamaterials, Soft Matter, Statistical Physics

\section{INTRODUCTION}

Single-degree-of-freedom (d.o.f.) mechanical structures are attractive in a range of fields, as almost any force will actuate that specific designed mode. Much like an umbrella or a folding chair, such "self-folding" structures can be reliably deployed even in uncertain environments with unreliable actuation forces. This principle has found wide use in kinetic or deployable architecture, heart stents, microelectromechanical systems (MEMS), sensors, and robots on a range of length scales [1-4]; recently, self-folding origami has become a popular framework for such applications [5-10].

The self-folding approach is similar in spirit to other bottom-up methods such as self-assembly of particles [11] and self-folding of polymers [12]; these methods exploit careful programming of interactions to allow for careless actuation at deployment. However, in these other selfactuating frameworks, the interactions needed for the desired assembly or folding inevitably create many other "distractor" states (e.g., kinetic traps in self-assembly [13-15] or in protein folding [12,16,17]), necessitating more care at deployment than one would naively expect.

Here, we show that it is difficult to fold self-folding origami (a thin sheet precreased to allow only a single folding motion) because of a similar inevitable proliferation of distractor folding branches. The distractor branches, shown schematically in Fig. 1, meet at a bifurcation at the

Published by the American Physical Society under the terms of the Creative Commons Attribution 4.0 International license. Further distribution of this work must maintain attribution to the author(s) and the published article's title, journal citation, and DOI. flat state, but they are dead ends since they are of zero energy only to linear order. The number of distractors grows exponentially with the size of the sheet, and consequently, most spatial distributions of folding forces will actuate a distractor [Figs. 1(c) and 1(d)]. As a result, despite having only one extended d.o.f., self-folding crease patterns require multiple actuators placed at carefully chosen spatial locations for successful actuation.

We trace the origin of distractors to frustrated loops of vertices, each of which can fold along one of two branches. Such frustrated loops create a glassy energy landscape for the sheet around the flat state, i.e., a landscape with an exponential number of local minima corresponding to the distractors. Material properties are expected to modify the precise details of this landscape, yet will not change its fundamental glassy nature. Successful folding must be seeded by actuation at a carefully chosen set of creases that picks out the ground state of the glassy landscape, much like with protein folding [17-20] and other satisfiability problems [21]. We find that the spatial arrangement of actuators needed can be understood heuristically in terms of unfrustrated "folding islands," the largest subpattern containing a given actuated crease that will fold when cut out of the full pattern.

In this way, our work shows fundamental limits to the programmability of self-folding sheets due to an inevitable glassy landscape of undesired states. In conjunction with similar limits in other bottom-up approaches like selfassembly [11] and self-folding polymers [12], our work adds to a common picture of glassiness intrinsic to bottomup methods based on frustrated and disordered interactions, independent of the details of specific implementations. In addition, our results provide a practical means of understanding where to place active creases. For example, 
(a)

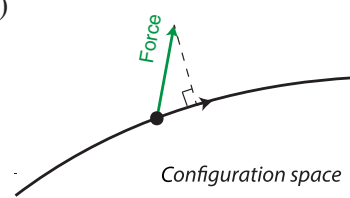

Single degree of freedom

(c)

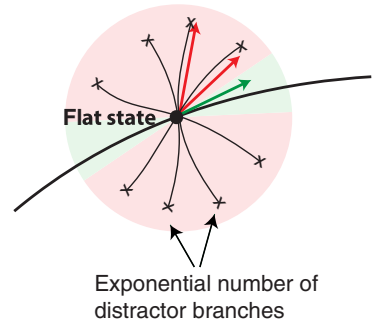

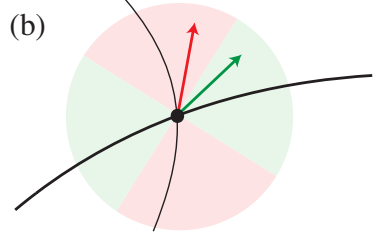

Branched degree of freedom

(d)

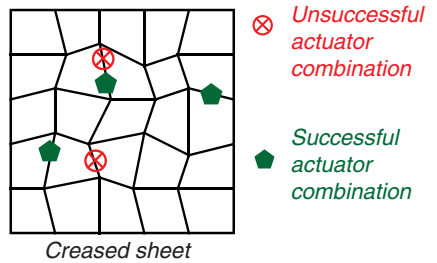

FIG. 1. (a) Structures designed with only one folding motion ("mechanisms") are thought to be easy to control since any applied force not exactly perpendicular to that motion will actuate it. (b) However, if a mechanism has a branched d.o.f. (bifurcation), the applied force (green) must make a smaller angle with the desired branch than with the undesired branch. (c) We show that programming a stiff sheet with one folding motion inevitably creates an exponential number of other dead-end distractor branches that are of zero energy only to linear order. The applied force needs to be highly aligned with the desired folding motion in order to avoid the distractors. (d) Consequently, we must actuate multiple creases in a carefully selected combination (green) to successfully fold a self-folding crease pattern.

in hydrogels or shape memory alloys, one must choose the active hinges; our theory predicts which combination of hinges would be successful and even predicts that sometimes, adding a new active crease (aiding in the right direction) to an existing successful actuation can in fact prevent folding.

Our results on glassiness and the difficulty of physically folding origami superficially resemble earlier works, such as Bern and Hayes's classic result on nondeterministic polynomial (NP) hardness of flat-foldability [22] and others [6,23-25]. However, Bern and Hayes focused on the ordering of folds in multistage folding, also investigated later in Refs. [26-28]. Here, we focus on self-folding sheets with a single temporal stage. More significantly, many earlier works $[22,25]$ concern the computational difficulty in finding consistent global mountain-valley assignments (e.g., "forcing sets" [23,24]), while our work concerns whether the physics of folding can find a desired global mountain-valley assignment, taking into account physical effects such as mechanical advantage and energy landscapes that play no role in these earlier works. A recent work [29] considers similar actuation questions for single vertices and simple loops of vertices; in contrast, we use an energy model and focus on statistical results for large quadrilateral meshes with an exponential number of distractors.

\section{RESULTS}

\section{A. 4-vertex and chains of 4-vertices}

When a vertex with $n$ creases is folded, the $n$ dihedral fold angles $\rho_{i}, i=1, \ldots, n$ are related by three equations [30]. Thus, $n$-valent vertices with $n \leq 3$ will be completely rigid, while vertices with $n \geq 5$ have multiple d.o.f. 4-vertices are of special interest as they have precisely one d.o.f.

However, a crucial caveat to this Maxwell counting is that only two of the three vertex equations are independent when the vertex is laid out flat [29] (i.e., unfolded). Consequently, it was shown [31] that a generic 4-vertex has two distinct folding branches that meet at a bifurcation at the flat state (Fig. 2).

To see this quantitatively, we follow Tachi's use of rotation matrices $[32,33]$ to derive three constraint equations $T_{a}(\vec{\rho} ; \vec{\theta})=0, a=1,2,3$, associated with the vertex, where $\vec{\rho}$ are the fold angles at creases and $\vec{\theta}$ are the in-plane angles between creases (see Appendix B). We expand the constraints $T_{a}$ in a series in $\rho_{i}$ about the flat state $\vec{\rho}=0$ as $T_{a}(\vec{\rho})=C_{a}^{i} \rho_{i}+D_{a}^{i j} \rho_{i} \rho_{j}+\cdots$ (where repeated indices are summed over). Configurations that violate these constraints will have $T_{a}(\vec{\rho}) \neq 0$ and we can associate an energy $E_{\text {Vertex }} \equiv \sum_{a} T_{a}^{2}$ with these configurations. See Appendix B for more discussion on alternative choices of energy.

Such an energy of a general vertex configuration scales as $\|\vec{\rho}\|^{2}$. However, $C_{a}^{i}$ has rank 2 , giving a two-dimensional space of zero modes in the linear approximation about the flat state $\vec{\rho}=0$. The energy scales as $\|\vec{\rho}\|^{4}$ for folding modes in this linearized null space (with no bent faces). Figure 2(b) shows the energy for folding modes within the linearized null space as we fold to larger angles. We see that two special folding branches within the linearized null space have zero energy to all orders. Thus, a generic 4-vertex has a full two-dimensional vector space of zero modes at the flat state in a linear approximation, but only two onedimensional branches of zero energy upon nonzero folding. This is consistent with Maxwell counting, as one constraint is redundant at, but only at, the flat state.

The two folding branches differ qualitatively in the sign of their fold angles. Both branches satisfy the following rule $[34,35]$; three of the four creases must fold in a common orientation (say, valley fold) with the final oddone-out crease folding the other way (mountain fold). The final odd-one-out crease can be either one of the two creases whose neighboring angles add to less than $\pi$; see Fig. 2(a). This discrete choice gives rise to the two branches. Note that the two creases capable of being the odd one out are always adjacent.

\section{B. Branch selection through mechanical advantage}

When external folding torques $\tau_{i}, i=1 \ldots 4$, are applied to the creases of a 4-vertex and released, the vertex will relax into one of the two branches [Fig. 2(a)] with 
(a)
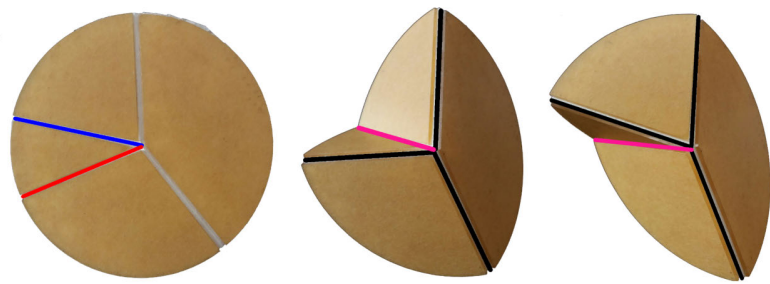

(1)

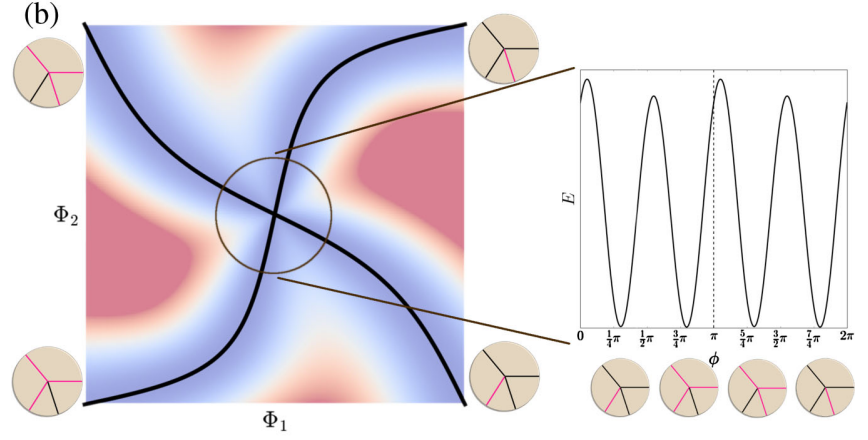

(c)

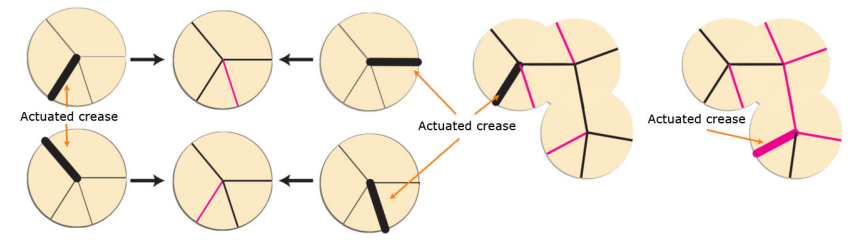

FIG. 2. Bifurcations for vertices and chains of vertices. (a) A single vertex has two distinct folding branches, in which three creases form a mountain fold (black line) and one becomes a valley fold (magenta), or vice versa. The distinct branches are identifiable by the odd-one-out crease that folds opposite to the rest, with the two choices marked by blue and red lines. (b) The two branches meet at a bifurcation at the flat state. $\Phi_{1}$ and $\Phi_{2}$ correspond to the two eigenvectors of folding angles that span the two-dimensional null space of the vertex. Given a folding amplitude (e.g., $\|\vec{\rho}\|=0.1$, we blow out the energy of any configuration on a circle of that radius, with two clearly identified zero-energy configurations (and their negatives). (c) When a selected crease is actuated, the vertex chooses the branch in which that actuated crease folds more relative to other creases (rule of mechanical advantage). Since the odd-one-out crease and its transverse crease tend to fold less than the other crease pair, the odd-one-out crease is generally adjacent to the actuated crease. (d) When $N$ vertices are linked together into an open-ended chain, the chain can fold in $2^{N}$ different folding branches. Given an actuated crease, the resulting MV data can be predicted by applying the branch selection rule of (c) to vertices in sequence, as each successive vertex is actuated through the crease linking it to the prior vertex.

corresponding folding angles $\vec{\rho}_{\alpha}, \alpha=1,2$. In the linear regime $\|\vec{\rho}\| \ll 1$, using our energy model [Eq. (1)] we find that computing the normalized dot product between the applied vector of torques ("applied force") $\vec{\tau}$ and the folding angles $\vec{\rho}_{\alpha}$ of the two branches identifies the actuated branch; the vertex will relax into the branch with higher dot product $\tau \cdot \vec{\rho}_{\alpha} /\|\vec{\tau}\|\left\|\vec{\rho}_{\alpha}\right\|$. This rule is equivalent to a selection based on mechanical advantage; when one crease is actuated, the vertex folds into the branch in which that crease's folding is larger relative to other creases (i.e., contributes more to the norm $\left.\left\|\vec{\rho}_{\alpha}\right\|\right)$.

Our mechanical advantage rule is based on a model energy landscape where the angular bisector of the two branches separates their attractor basins. In real material vertices, the dividing line between the attractors might be closer to one branch than the other (Appendix C); such complications do not change our results qualitatively. In contrast, the authors of a recent work [29] assumed that actuation might fail if the applied force has a positive dot product with any other available branch. In such a model, even applied forces perfectly aligned with a branch may be classified as incapable of evoking that branch, in contrast to energy-landscape-based models.

Our mechanical advantage rule can be restated as a heuristic in terms of mountain-valley (MV) choices. In either folding branch, the crease with odd-one-out MV state and its transverse crease fold less than the other pair of creases that share a common MV state [29] (To see this intuitively, consider the limiting case in which all in-plane angles are nearly 90 degrees and the vertex folds in half along one pair of creases with the same MV state; the other pair of creases barely fold at all.)

Combining this observation with the dot product rule, we conclude that when a single crease is actuated, the vertex will choose the branch in which the crease transverse to the control crease will fold with the same MV state [Fig. 2(c)].

This branch-picking rule is easily extended to chains or trees of vertices, as long as no loops are present. If we actuate at one select crease at a vertex in this chain, we can determine the branch choice at that vertex using the above rule and thus the MV state of all creases at that vertex. Any neighboring vertex is actuated by the creases connecting them. In the absence of loops, there is only one path from the controlled vertex to any other and hence the modepropagation rule unambiguously determines the branch choice at each vertex [Fig. 2(d)].

In this way, for any given actuated crease, the branch selection and propagation rule unambiguously selects one branch out of the $2^{N}$ bifurcated folding branches of an $N$ vertex chain. Thus, at least with idealized materials, a choice of branch can easily be made in the loopless case. In contrast, we will now show that patterns with loops, even if made from idealized materials, are intrinsically difficult to fold.

\section{Loops of vertices create glassy energy landscapes}

If 4-vertices are connected around a loop, we can no longer make an independent choice of folding branch at each of the vertices. For example, for a loop of four 4-vertices like that in Fig. 3(a), we can make independent branch choices for three of the vertices-say, for $V_{1}, V_{2}$, and $V_{3}$-which puts them in one of their zero-energy states [red or blue points in Fig. 3(b)]. The final vertex's folding branch is then completely determined because the states of 
(a)

(b)

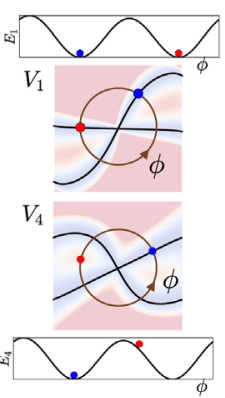

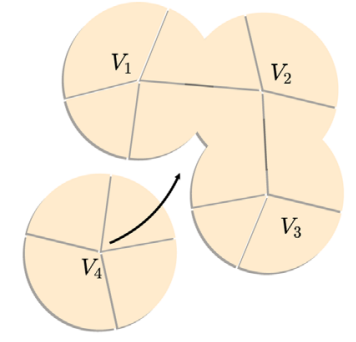

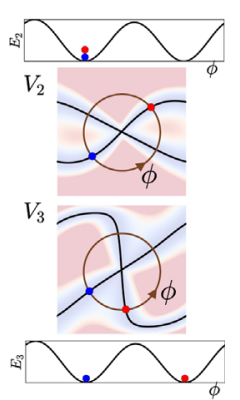

(c)
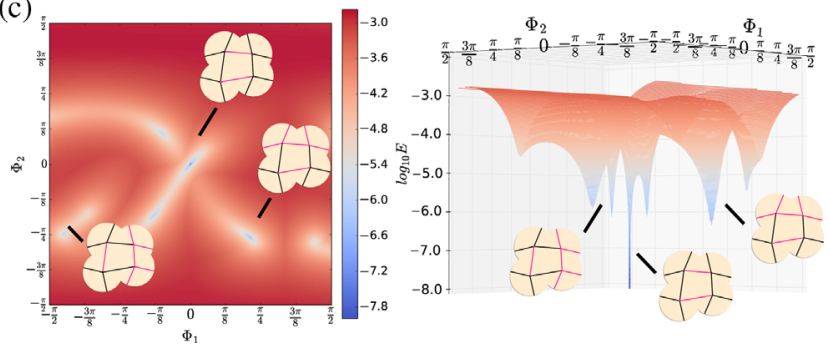

FIG. 3. Loops of vertices give rise to a glassy landscape. (a)-(b) When a chain of vertices is closed by adding a final vertex, the resulting branches are no longer of zero energy. For example, if vertices $V_{1}, V_{2}, V_{3}$ are at one of their two zero-energy states (red dots), $V_{4}$ 's folding state is constrained since the folding states of creases $V_{1}-V_{4}$ and $V_{3}-V_{4}$ are already set. The resulting energy for $V_{4}$ is generically not zero (red dot for $V_{4}$ ). We computed the energy in the four-dimensional linearized null space at a fixed norm $\|\vec{\rho}\|$. (c) The heatmap and surface plot show a two-dimensional projection onto the two top eigenvectors $\Phi_{1}$, $\Phi_{2}$ in the linearized null space.

two creases at $V_{4}$ are already determined (namely, creases $V_{3}-V_{4}$ and $\left.V_{4}-V_{1}\right)$. Generically, the resulting state for $V_{4}$ will not be of zero energy [36] [red dots in Fig. 3(b)]. We thus find that the resulting folding branch is of nonzero energy, unlike for chains of vertices.

Such thin sheet configurations with nonzero energy will also show face bending. We add a stiff face diagonal to each inner face in a crease pattern with face stiffness parameter $\kappa_{f}$ (see Appendix B for more details, specifically on the balance of stretching and bending). The energy model of a generic configuration with loops thus becomes

$$
E \equiv E_{\mathrm{Vertex}}+E_{\mathrm{Face}}=\sum_{a} T_{a}^{2}+\frac{1}{2} \kappa_{f} \vec{\rho}_{f}^{2},
$$

where $\vec{\rho}_{f}$ are the face bending angles.

Going through the $2^{3}=8$ independent branch choices for $V_{1}, V_{2}, V_{3}$ (which then determine the state of $V_{4}$ ), we should expect to generically find eight branches of nonzero energy. In fact, these folding branches are of zero energy to quadratic order but of nonzero energy at next order; i.e., the energy of these branches scale as $\kappa \rho^{4}$ with $\kappa \neq 0$. In contrast, $\kappa=0$ for all the $2^{N}$ folding branches of a chain of vertices. To gain more intuition about these branches and their energies, we fixed the overall folding magnitude $\|\vec{\rho}\|$

for a single four-loop and computed the energy as a function of the angular directions in $\vec{\rho}$ space. A twodimensional projection is shown in Fig. 3(c), where each branch shows up as a local minimum with depth proportional to $\kappa$. (See Appendix B for more details, including accounting for finite face bending and stretching energies at different $\|\vec{\rho}\|$.)

Thus, we find that loops of vertices have a glassy folding energy landscape, much like a spin network with frustrated loops [37], and unlike trees or chains of spins.

A desired branch's energy can be made arbitrarily low or even zero to all orders in folding by fine-tuning in-plane angles using "loop" equations $[38,39]$. While the design process can make a desired folding branch be the ground state of the landscape, it does not change the glassy attractor structure shown in Fig. 3(c); see Appendix A (Fig. 6) for comparison. Different actuated creases initialize the folding process in different parts of the glassy landscape; folding then involves flowing downhill to a local minimum. Hence, actuating a desired branch in such a landscape can be difficult in the presence of a multitude of distractor branches.

\section{Large patterns: Number, attractor size of distractors}

Large patterns made of many 4-vertices contain many loops and the number of distractor branches grows rapidly. We generated quadrilateral meshes of random geometry made of $\sqrt{A} \times \sqrt{A}$ vertices, folded each mesh with random applied forces $\vec{\tau}$, and allowed it to relax into a local energy minimum until no more new minima were discovered. In this way, we determined the following landscape properties:

(a) The total number of distinct branches $N_{\text {branches }}$ for a given quadrilateral mesh grows exponentially with the size of the mesh, with the precise number of minima depending on the distance $\|\vec{\rho}\|$ from the flat state at which folding is stopped [Fig. 4(a)].

The increase in the exponential number of minima with folding distance arises because of the well-studied relationship between stretching and bending in thin sheets [39-41]. As discussed in Appendix B, close enough to the flat state, face bending and stretching energies are comparable. In this regime, as suggested by Fig. 3(a), choosing the states of three vertices around the loop strongly constrains the state of the fourth vertex. As one folds more, face bending becomes less expensive than stretching for thin sheets. Consequently, constraints on the fourth vertex weaken, revealing a larger (but still exponential) number of branches at larger $\|\vec{\rho}\|$, as shown in Fig. 4(a).

Note that while our numeric results unambiguously show exponential growth for all the $\|\vec{\rho}\|$ shown in Fig. 4(a), we find that the precise form cannot be numerically determined with confidence, despite $N_{\text {branches }}$ varying over 2.5 orders of magnitude. Note that in the limit of free face bending, our self-folding mesh is transformed into the fully triangulated patterns studied recently in Ref. [42]. 


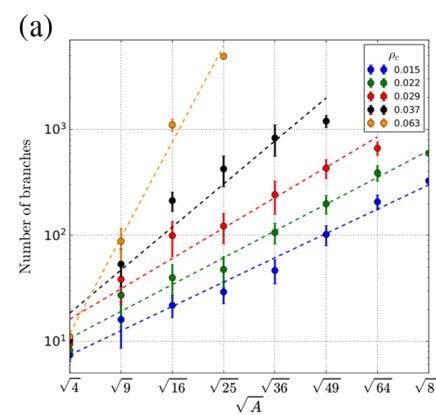

(b)

FIG. 4. Large patterns have an exponential number of branches (i.e., minima) of decreasing attractor size. We characterized the landscape by sampling random quadrilateral meshes of size up to $A=81$ vertices and folded each one with random torques until no new stable branches were found $\left[\kappa_{f}=10^{-6}\right.$; see Eq. (1)]. (a) Quadrilateral meshes show an exponential number of distinct folding branches (i.e., local minima) in their energy landscape; the precise scaling depends on the total extent of folding $\|\vec{\rho}\|$, reflecting the relative importance of bending and stretching energies (Appendix B). (b) The size of attractor basins around different branches for a fixed pattern does not exceed $15 \%$ of the total space for a $4 \times 4$ mesh.

(b) The attractor size of each distractor branch (taken to be the fraction of random actuation forces that actuate the branch) is generally small; see Fig. 4(b). The largest attractor for the $4 \times 4$ mesh sampled is only $\approx 15 \%$; i.e., only $15 \%$ of random torques will actuate that branch. Most branches have far smaller attractor basins. The typical attractor size is expected to drop sharply with $A$. Data for small patterns up to $A=64$ suggests a power law dependence of the mean attractor size.

\section{E. Actuation of large loopy patterns}

How many creases need to be actuated-and which ones-to pick the desired branch in a landscape with an exponential number of other minima? Such landscapes arise in diverse areas of physics for the same reasonfrustrated disordered interactions - and are often referred to as "complex" or "glassy" [12,17,21,37,43].

To answer this question for self-folding origami, we study a random pattern with a chosen branch, shown in Fig. 5(a). Since the crease locations at which folding torques are applied can be better controlled than the precise magnitude of torques in many applications [1], we applied folding torques of fixed magnitude to different randomly selected subsets of creases. The applied torques were always of the correct sign (mountain or valley) needed at that crease for the chosen branch. As seen in Fig. 5(b), actuators are needed on 18 out of a total of 60 creases to have a $50 \%$ probability of folding the pattern.

For applications where the precise torque magnitudes can be controlled in addition to location (as explored recently in [29]), we must characterize how closely the applied vector of torques must align with the folding angles of the desired branch (see Fig. 1). We present such results on dot products in Appendix D.

Requiring a large number of actuators or precise control of torque magnitudes defeats the purpose of designing a single-d.o.f. mechanism; it is hard to call a system requiring such delicate control "self-folding".

How then can self-folding origami be folded with a minimal number of actuators? A lesson can be drawn from similar glassy landscape search problems in models of protein folding (e.g., Levinthal's paradox $[17,19,20,44])$ and related NP-hard satisfiability (SAT) problems $[21,45]$ that vary from the traveling salesman problem to Sudoku [46]. A common element in these satisfiability problems is that random seeding of the search for the global minimum leads to repeated backtracking after reaching local minima, both in the context of computer algorithms (as the Davis-PutnamLogemann-Loveland (DPLL) algorithm for k-SAT [21]) or for physical dynamics (as in protein folding) [45]. However, careful seeding of the search-e.g., if the right boxes are filled in first in Sudoku [46] or if the right parts of the protein are folded first - can greatly reduce or even eliminate backtracking [21] before reaching the global minimum.

Correct seeding is even more important for origami since folding is assumed to happen at "zero temperature" (e.g., without any noise or fluctuations). As a result, the structure cannot backtrack out of a local minimum as in the case of nonzero temperature SAT problems [45].

\section{F. Folding islands}

To understand the role of frustration and seeding in the origami context, we must consider both the branch selection rule and the effect of loops. Even in the absence of loops, when an actuated vertex is folded into its desired branch, the MV state propagated to a target vertex, as shown in Fig. 2(d), can disagree with the desired folding branch at the target vertex and thus fold it incorrectly.

The situation is complicated by the presence of loops since a target vertex can be reached from a control crease by multiple paths. The mechanical advantage heuristic applied to different paths, which reach a particular vertex from different directions, may not be consistent. For example, different paths might imply different folding branches for the target vertex. Thus, in the presence of loops, the mechanical advantage heuristic (or any other such pathbased heuristic) is not sufficient for folding all vertices into desired branches.

As a result, while a designed folding branch guarantees a globally consistent configuration of vertex branch choices [e.g., blue dots in Fig. 3(b)], such a global configuration may be difficult to reach using the local MV propagation rule in Fig. 2(c) from a single actuator. Hence, successfully folding a large pattern in a desired branch can require actuating multiple creases at the same time.

A clue to finding good sets of actuators is seen in Fig. 5(c): When actuated at a single crease, the obtained (undesired) 
(a)

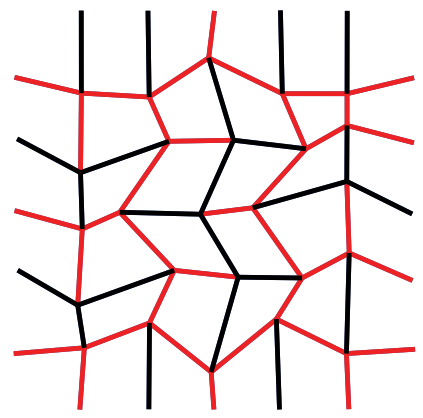

(d)

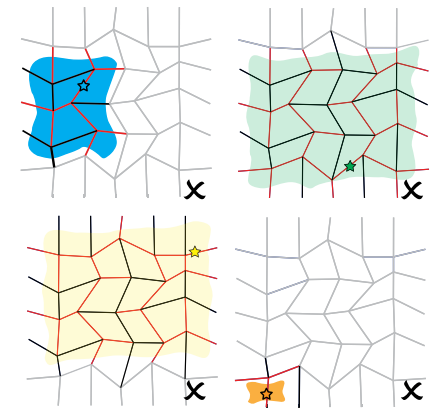

(h)

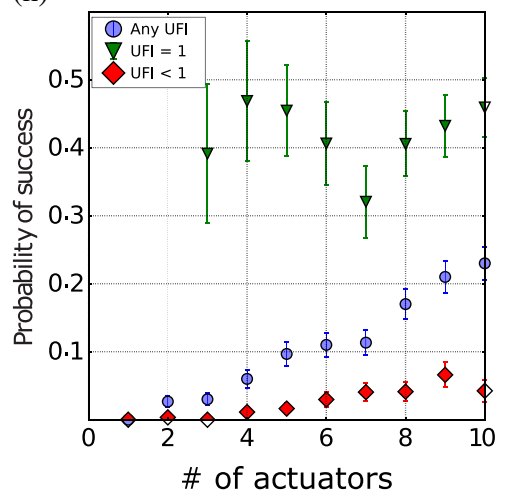

(e) (b)
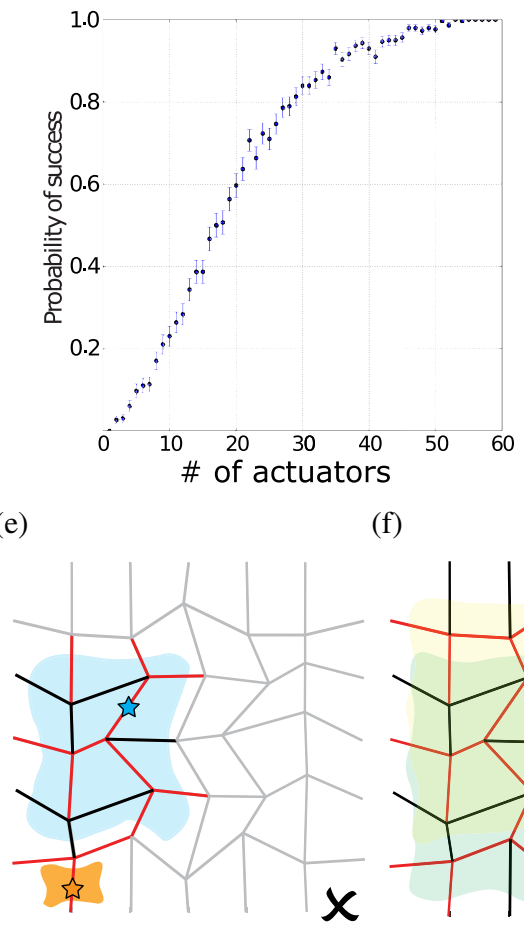

(i)

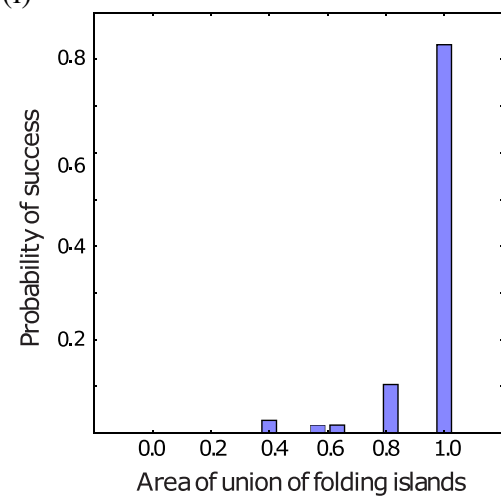

(c)

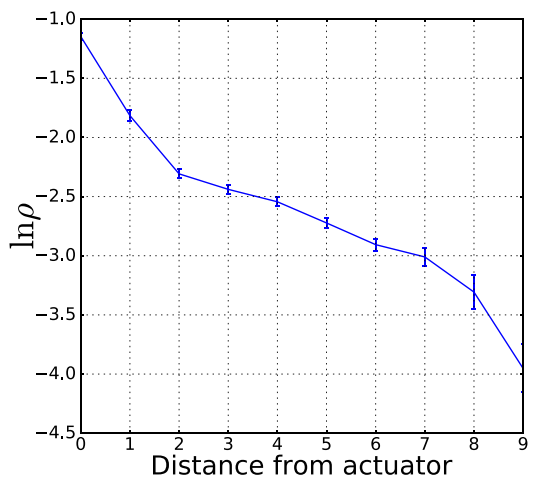

(g) (f)

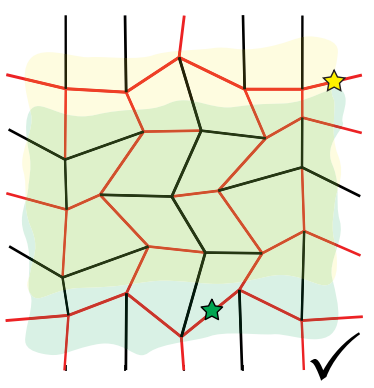

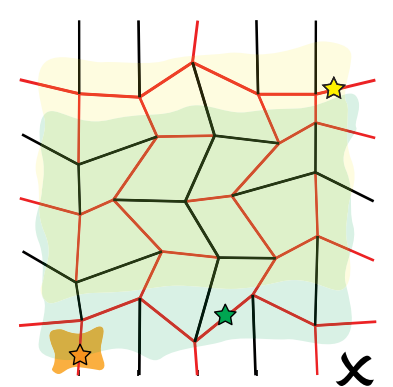

(UFI)

FIG. 5. Spatial distribution of actuators determines folding success. (a) A $4 \times 4$ quadrilateral mesh pattern, with their designed soft branch indicated by line colors (black for mountain, red for valley). (b) If standard actuators are placed on randomly chosen creases of the pattern, at least 18 actuators ( $\sim 30 \%$ of creases) are needed to have a $50 \%$ chance of successful folding. (c) If just one crease is pressed, the resulting branches typically have a decreasing folding magnitude for creases away from the actuated crease. (d) The folding island of a crease is the largest subpattern that folds correctly when cut out from the full pattern and actuated at that crease. The area of the union of folding islands (UFI) relative to the entire pattern provides a simple design heuristic. (e) Actuated crease sets with UFI < 1 generally do not successfully fold the pattern while (f) actuators with UFI $=1$ are successful. (g) However, successful actuation can sometimes be ruined by actuating an additional crease (orange) with a small folding island. (h) Actuated crease sets of a given size are dramatically more likely to fold successfully if their UFI $=1$ (green) rather than UFI $<1$ (red). (i) Using the data in (b), we find that UFI is a sharper predictor of success than the number of actuators. (j) Folding islands also explain a counterintuitive effect where successful actuation can sometimes be ruined by actuating an additional crease (orange) with a small folding island. The obtained branch is different than the designed one primarily in creases close to the "bad" actuator (thick lines).

branch is generally "localized," where folding amplitudes near the actuated crease are larger than those far away from it. This suggests that globally desired folding might be achieved with cooperative local actuators, each of which fold their local neighborhoods correctly.
To find the number of actuators needed for successful actuation of a desired branch, we identify unfrustrated subpatterns called folding islands. We define the folding island of a crease (with respect to a desired folding branch) as the largest contiguous region of the pattern that will fold 
in the desired branch, if that region is cut out and actuated at the chosen crease. For computation of the folding islands, see Appendix E. Figure 5(d) shows that folding islands for different creases can vary greatly in size and generally do not cover the whole pattern. While folding islands can be approximately deduced using the simple MV propagation rule in Fig. 2(c), the exact shape depends on the precise in-plane angles.

These considerations suggest a heuristic necessary condition for a set of actuated creases to fold a pattern; the union of their folding islands should cover the whole pattern. If not, as in Fig. 5(e), when folding reaches the boundary of a folding island, folding will jam in a highenergy distractor branch as vertices outside the union of islands will fold incorrectly. On the other hand, the two actuators shown in Fig. 5(f), whose folding islands together cover the entire pattern, successfully fold the pattern.

Folding islands provide a new perspective on why randomly placed actuators [Fig. 5(b)] were poor at folding the pattern. In Fig. 5(h), we went through the different actuated crease sets used in Fig. 5(b) and computed the area of the union of folding islands (which we denote UFI, defined as the fraction of all creases belonging to the union) for each set. We see, for example, that a set of five actuators is $60 \times$ more likely to fold the pattern if it has UFI $=1$ rather than UFI $<1$. Similarly, Fig. 5(i) shows all the data in Fig. 5(b), but plotted against UFI instead of the number of actuators. These results show the union of folding islands, and thus, spatial placement of actuators is a much better predictor of folding success than the number of actuators (We find a few cases of successful actuation, e.g., at UFI $=0.8$ when the folding islands cover most vertices.) In particular, the condition UFI $=1$ eliminates many spatial arrangements of actuators that are nearly guaranteed to fail.

Folding islands also shed light on a counterintuitive phenomenon shown in Fig. 5(g). While the two actuators in Fig. 5(f) can successfully fold the pattern, adding another actuator with a very small folding island, as in Fig. 5(g), can stop the previously successful folding. Figure 5(j) shows the resulting undesired branch, which is different than the desired branch in the bold creases. Thus, the interaction between the green and orange actuators leads to misfolding, mostly in proximity to the orange actuator (just outside of its small folding island), consistent with the falling influence of actuators with distance as shown in Fig. 5(c). Such effects reduce the probability of success in Fig. 5(g) when UFI $=1$ to be less than 1. Predicting these subtle competitions between different control creases requires knowledge of the precise in-plane angles of the pattern, and we are unable to formulate a strictly necessary and sufficient condition for successful folding without full pattern information. Nevertheless, identifying the folding islands provides a useful design heuristic to greatly reduce the number of actuators needed, as seen in Figs. 5(h) and 5(i). See Appendix $\mathrm{E}$ for more on how folding islands can be incorporated into an algorithmic framework for actuator placement that is vastly more feasible than blind search (experimental or in simulations) through all combinations of actuators.

\section{DISCUSSION}

Sheets with crease patterns designed to exhibit exactly one folding behavior are nevertheless difficult to fold. We traced this difficulty to the fact that stabilizing one folding behavior using frustrated interactions between binary d.o.f. (bifurcated origami vertices [30,31]) inevitably stabilizes an exponential number of other distractor behaviors, i.e., a complex or glassy landscape [43]. Thus, our results establish fundamental limits on the programmability of energy landscapes for sheets, paralleling similar limitations in other bottom-up approaches such as self-assembly of particles [11] and self-folding of polymers [12], as well as classic NP-hard satisfiability (SAT) problems $[21,45]$.

Self-folding with real materials can introduce other complications, specific to those realizations, that make folding more difficult than described in our paper. In Appendix C, we explore several other models of selffolding sheets, incorporating stiffness of creases, variable bending vs stretching energy of sheets using COMSOL, and manufacturing error in crease placement. We find that the statistical properties of the glassy landscape remain unchanged. Thus, our work points to a fundamental glassy difficulty that is intrinsic to self-folding, reliant only on frustrated interactions between bifurcated 4-vertices-and hence must be faced by any material realization.

We saw that many actuators are needed to successfully fold self-folding sheets, if their locations are randomly chosen. However, carefully choosing the set of actuated creases can reduce their number dramatically. We interpreted successful combinations in terms of unfrustrated subpatterns called folding islands that successfully fold when cut out of the full pattern. The connection to protein folding and other NP-hard problems drawn here suggests other ways forward, including temporal staging, folding funnels, and chaperoned folding [12,21].

Recent self-folding origami applications vary greatly in the materials used and in actuation mechanisms for active hinges, including electric [1], optical [47], thermal [48], and chemical (pH) [49] methods. In many applications, energy can be selectively input to specific creases, e.g., by controlling the electric current to shape-memory polymer hinges $[27,50]$ or light input to hydrogels [36]. Our work suggests which combinations of creases should be given energy input for successful folding, even showing how adding an actuator can ruin successful folding [Fig. 5(h)]. Going beyond self-folding patterns, our considerations also apply to each temporal stage of multistage sequential folding patterns [26-28].

The folding difficulty described here and the resulting need for careful actuation mathematically applies only at 
the flat state; but since the energy barriers between branches grow more slowly with folding for a softer sheet, careful actuation needs to be maintained until a larger folding angle for soft sheets.

Recent experiments on controlled repeated crumpling and extension of sheets suggest an inability to refold along existing creases, leading to the formation of new creases [51]. While the 4-vertex patterns studied here are not good models of crumpled soft paper with significant face bending, our results do suggest that the difficulty of refolding a crease pattern, and thus the propensity to create new creases, grows with the softness of the sheet and when unfolded closer to the flat state.

\section{ACKNOWLEDGMENTS}

This work was primarily supported by the University of Chicago Materials Research Science and Engineering Center, which is funded by National Science Foundation under Award No. DMR-1420709. This work was also partially supported by NSF Grant No. CBET 1336489. We acknowledge the University of Chicago Research Computing Center for computing resources. We thank William Irvine, William Jacobs, Yoav Kallus, Christian Santangelo, Cristopher Moore, and Thomas Witten for insightful discussions.

\section{APPENDIX A: DESIGN OF FOLDING BRANCHES AND THE ENERGY LANDSCAPE}

An origami pattern containing loops made of 4-vertices inevitably has many folding branches of finite energy. For a generic pattern of some particular topology (e.g., a "quad" made of four 4-vertices in a loop), the folding branches have nonzero energies distributed over a wide range when folded with the same magnitude $\rho \equiv\|\vec{\rho}\|$ [Fig. 6(a)]. However, all these energies scale as $E \sim \rho^{4}$, since the folding branches are within the null space of the linear part of the expression for energy.

For many applications, one requires softer folding branches that can be folded to a nonlinear extent (large $\rho$ ) with a small input energy. Designing such soft folding branches can be accomplished by fine-tuning in-plane angles using loop equations $[38,39]$. The process entails picking a branch and modifying the geometry of the pattern to make that branch gradually softer. Each successive loop equation, when solved exactly, changes the scaling of the folding energy for the designed branch,

$$
E(\vec{\rho}) \sim \rho^{2+4 n},
$$

where $n$ loop equations are solved in sequence.

We can thus design a specific branch of the pattern to be as soft as needed, but what becomes of all the other folding branches of the pattern? We find that the other branches, for which no loop equations are solved, remain qualitatively
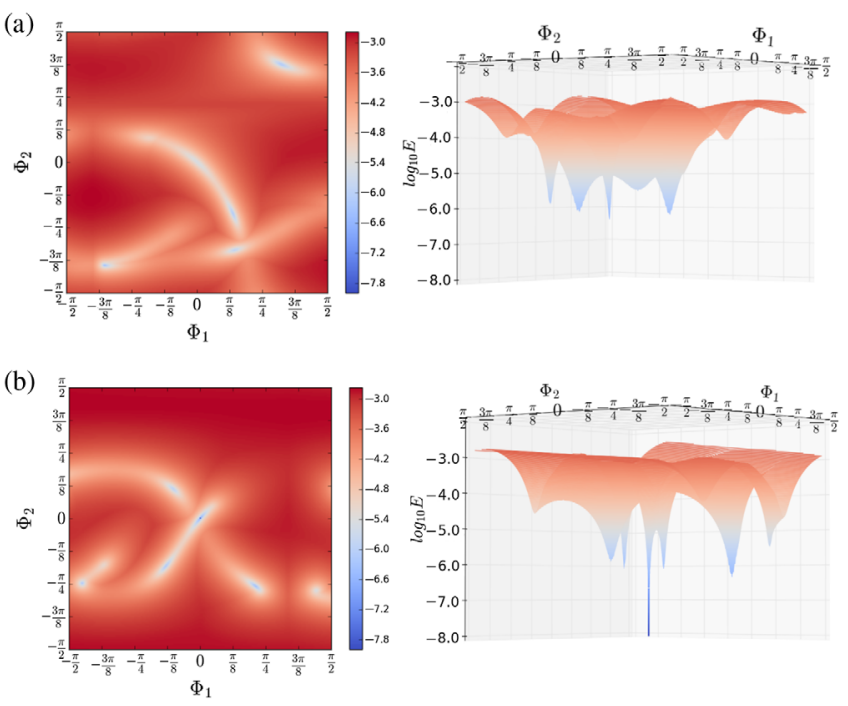

FIG. 6. Energy landscape of a quad loop pattern at a fixed norm of folding angles $\|\rho\|$. (a) A generic quad loop pattern has three to seven folding branches seen as minima in the energy landscape. Left: Heat map. Right: Corresponding threedimensional surface. Generic minima have energies that scale as $E \sim \rho^{4}$. (b) By solving loop equations [38,39], we can design one specific folding branch to be qualitatively softer than all others (identified as the much deeper minimum). The rest of the branches retain their original energy scaling and thus become distractors in the energy landscape.

the same. Their number in general does not change, and neither does their energy. As their energy still scales with $\rho^{4}$, we are left with a distinct energy landscape, where the one designed branch is qualitatively softer than all of the rest [Fig. 6(b)]. We thus call the other branches distractors, as they correspond to high-energy minima in a glassy landscape, with energy much higher than the ground state (i.e., the designed branch).

A remarkable fact about the distractor branches is that they do not have to comply with single-vertex rules. One might find a distractor branch in which a few vertices have "illegal" (non-Kawasaki-Justin) configurations, e.g., four valleys or mountain, two valleys or mountains, etc. Clearly, vertices with such configurations must contribute to the energy of the entire pattern, but they can do so within the linearized null space of the pattern. It is notable that all designed soft branches must be "legal" at the single vertex level. The energy of any configuration containing illegal assignments for any vertex scales at least as $E \sim \rho^{4}$.

\section{APPENDIX B: ENERGY AND VERTEX CONSTRAINTS}

A single 4-vertex has two zero-energy folding branches that extend to arbitrary overall folding magnitude $\|\vec{\rho}\|$. These modes cost no energy as the corresponding choices of $\vec{\rho}$ exactly satisfy the vertex constraints. 
Vertex constraints are derived for any closed vertex by considering a condition such that the vertex does not tear open when folded $[32,33]$. A small disk surrounding the vertex configuration is defined by the folding angles $\rho_{i}$ and the in-plane angles between the creases $\theta_{i}$. One can "walk around" the edge of the disk around the vertex and eventually return to the same point only if the vertex is not torn anywhere. This motion around the vertex consists of rotating with the $\theta_{i}$ angle about the central axis of the vertex and then rotating about the dihedral angle $\rho_{i}$ until one returns to the original position. If one orients the current vertex face such that the face occupies the $x y$ plane and the crease is on the $x$ axis, these two rotations can be expressed as [32,33]

$R_{i}=\left(\begin{array}{ccc}1 & 0 & 0 \\ 0 & \cos \rho_{i} & -\sin \rho_{i} \\ 0 & \sin \rho_{i} & \cos \rho_{i}\end{array}\right)\left(\begin{array}{ccc}\cos \theta_{i} & -\sin \theta_{i} & 0 \\ \sin \theta_{i} & \cos \theta_{i} & 0 \\ 0 & 0 & 1\end{array}\right)$.

In general, $R_{i}=A_{i} B_{i}$, where $A_{i}$ is a rotation matrix about an axis along crease $i$ by angle $\rho_{i}$, while $B_{i}$ is a rotation matrix about an axis perpendicular to face $i$ by angle $\theta_{i}$.

The condition that the vertex is not torn becomes

$$
\prod_{i} R_{i}=I
$$

where the product is taken over all creases and faces $i$, and $I$ is the $3 \times 3$ identity matrix. Equation (B2) can be shown to be equivalent to three independent equations for the offdiagonal upper matrix. Crucially, these are three nonlinear constraints relating the folding values $\rho_{i}$ around the vertex. A 4-vertex is a one-d.o.f. object, as three equations relate its four folding angles.

Near the flat state, all $\rho_{i} \approx 0$, and the matrices of Eqs. (B1)-(B2) become essentially two-dimensional rotation matrices about an axis perpendicular to the flat vertex; hence, one vertex constraint is lost. The preceding considerations apply to all vertices constructing the pattern.

As shown in the main text, the constraints can be expanded about any configuration, in particular the flat state $\vec{\rho}=0$ :

$$
T_{a}(\vec{\rho})=C_{a}^{i} \rho_{i}+D_{a}^{i j} \rho_{i} \rho_{j}+\ldots
$$

Violation of these vertex constraints can be interpreted as a stretching energy at that vertex, $E_{\mathrm{Vertex}}=T_{a}^{2}$. Figure 7 illustrates the vertex energy in this model. Random configurations of $\vec{\rho}$ for which $C_{a i} \rho^{i} \neq 0$ are characterized by an energy that grows quadratically in $\rho$. Configurations existing in the two-dimensional linearized null space of the vertex have a scaling $E \sim \rho^{4}$. Large patterns made of 4-vertices have a high-dimensional linearized null space where the energy scales like that of a vertex. However, we

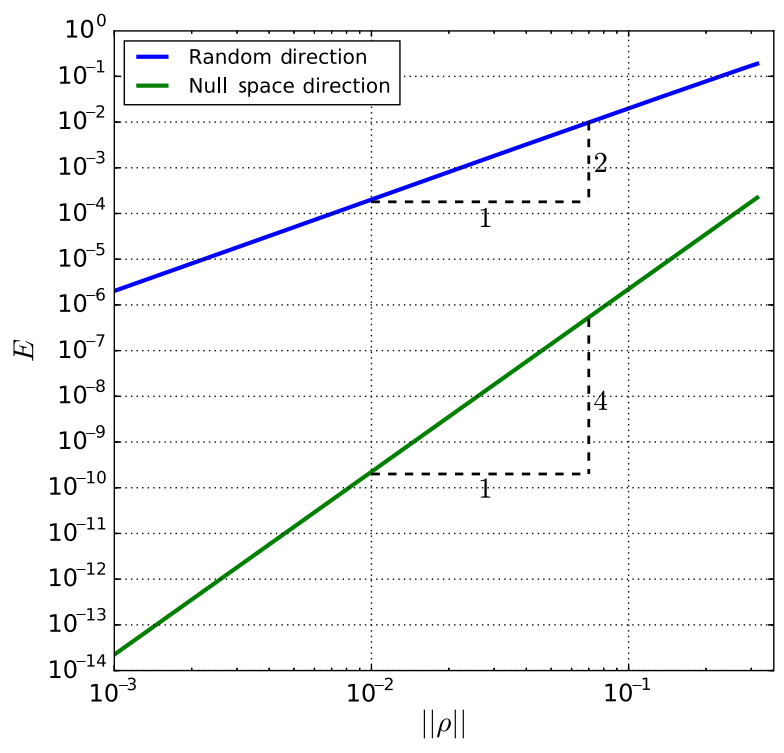

FIG. 7. Stretching energy scaling in a 4-vertex $E(\|\vec{\rho}\|)$ depends on the direction of $\vec{\rho}$. For random directions, we have $E \sim \rho^{2}$, while for directions within the linearized null space of the system, $E \sim \rho^{4}$. Only two special directions (not shown) exist for which $E=0$ to all orders in folding.

show in the main text that patterns with loops are frustrated, such that in general no zero-energy folding branches exist.

In addition to vertex energy, when vertices form loops, one also expects face bending in thin sheets [40,52]. We model such face bending by adding stiff face diagonals to each inner face; that is, we add face diagonals with torsional springs on them of stiffness $\kappa_{f}$ and rest angle $\rho_{f}=0$. Putting these together, our energy model is

$$
\begin{aligned}
E(\vec{\rho}) & \equiv E_{\text {Vertex }}+E_{\text {Face }} \\
& =\sum_{a} T_{a}^{2}+\frac{1}{2} \kappa_{f} \sum_{i \in \text { faces }} \rho_{i}^{2},
\end{aligned}
$$

with $\kappa_{f}$ a proper dimensional face stiffness factor. As a sheet is made thinner, the bending modulus is reduced relative to stretching [40,41], effectively reducing $\kappa_{f}$.

Every folding branch of generic looped patterns balances stretching (i.e., vertex) and bending (i.e., face) energies, with relative importance depending on the folding amplitude $\rho$. To illustrate the importance of the different $\rho$ scaling of the two energy terms, we counted the number of branches for quadrilateral meshes of different sizes, at different values of folding magnitude $\rho_{c}$ (Fig. 8).

Note that the stretching term scales as $\rho^{4}$ (for branches in the linearized null space), while the bending term scales as $\rho^{2}$. Thus, at smaller folding magnitudes bending of faces is expensive and suppressed. In contrast, at large folding magnitudes, stretching is more expensive and faces can bend much more freely. As explored in earlier work [39], the number of branches of origami patterns 


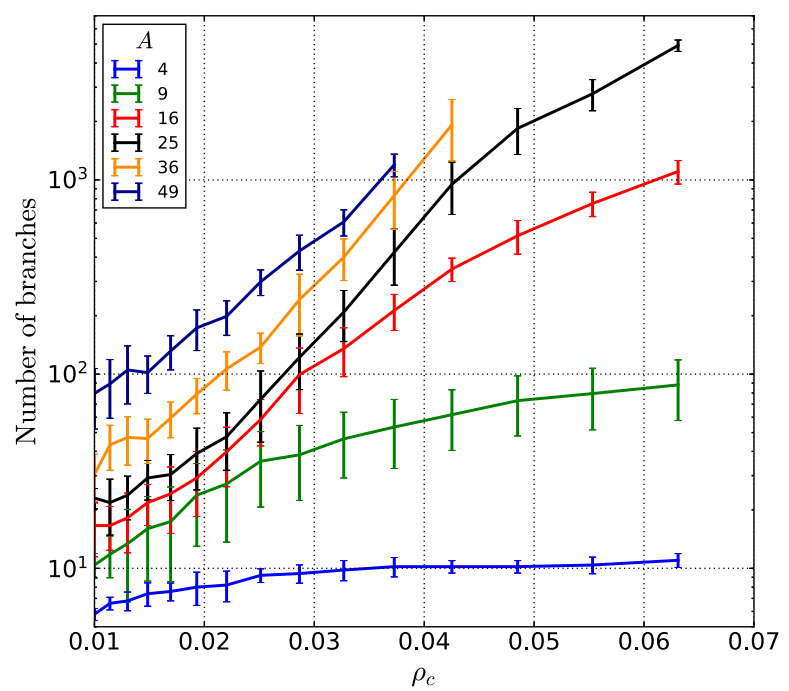

FIG. 8. Number of folding branches for patterns of different sizes, folded to magnitude $\left\|\vec{\rho}_{c}\right\|$. At large folding angles, the same patterns have many more folding branches due to the lesser importance of face bending compared to stretching. In the main text, Fig. 4(a) shows vertical slices of the same data, illustrating the exponential size scaling of the branch number $\left(\kappa_{f}=10^{-6}\right)$.

scales exponentially with the ratio of face bending to crease folding, consistent with these results.

Finally, while these models quantify the violation of constraints (using vertex energy and face bending, respectively) and a glassy landscape emerges in this context, the results hold for arbitrarily stiff sheets. However, these results cannot be derived directly for strictly infinitely stiff sheets, a mathematical idealization that assigns infinite energy to all non-zero-energy folding configurations. Consequently, that mathematical limit misses the glassy landscape that exists for arbitrarily stiff sheets. In this sense, softness is a singular perturbation and strictly infinitely stiff sheets are not a good approximation of realistic sheets, no matter how stiff, for the question of actuation.

\section{Folding method}

When external folding torques are applied to creases of a pattern, we fold the pattern by accounting for both the external and the internal forces generated by the energy model above [Eq. (1)].

Given an external folding torque $\tau_{i}$ applied to crease $i$, the folding of an "overdamped" but unconstrained crease follows $\gamma \dot{\rho}_{i}=\tau_{i}$, with $\gamma$ the stiffness of the crease. In selffolding patterns, folding motions are one-dimensional such that there exist preferable configurations $\rho_{i}$. This notion is encoded by the energy model presented above, as unfavorable configurations require more bending of the faces. Thus, if left on its own, the pattern will change its configuration introducing internal torques $\gamma \dot{\rho}_{i}=-\left[\partial E(\vec{\rho}) / \partial \rho_{i}\right]$.

These considerations allow construction of a folding algorithm, implemented as an ordinary differential equation (ODE) system:

$$
\gamma \frac{d \rho_{i}}{d t}=\tau_{i}-\frac{\partial E(\vec{\rho})}{\partial \rho_{i}}
$$

In practical computation, we usually start the solution from the flat state $\rho_{i}=0$ and solve this system with a fixed external torque $\tau_{i}$ until one of the dihedral angles reaches a certain predefined value (say, $0.5 \mathrm{rad}$ ). The equations are solved using the MATLAB ODE solver.

\section{APPENDIX C: EFFECTS OF MATERIAL PROPERTIES AND IMPERFECTIONS}

So far, we have considered a simplified model for selffolding origami made of stiff elastic materials. Bending energy is represented by stiff face diagonals, while all stretching energies are focused at the vertices as constraints. Creases themselves are free folding with no stiffness. Our model is a good approximation for self-folding origami in which the sheet is very thin and faces do not bend too much.

However, real origami made of realistic elastic materials is more complicated. This appendix illustrates how changing the energy model to incorporate the imperfections and complications of real materials-e.g., manufacturing error, crease stiffness, nonlocalized bending and stretching, finite thickness of sheets-modifies the configuration energy landscape.

The main results are that while these complications can indeed change details of the landscape, such as the precise number and energy of the minima, the underlying exponential structure and statistical properties of the landscape remain unchanged since they arise from the bifurcation of the 4-vertex. We thus conclude that while folding in specific real materials can face additional complications than those described here, our results point at foundational hurdles intrinsic to the concept of self-folding that must be faced by any material.

\section{Stiff creases}

So far we modeled idealized origami patterns in which the faces are stiff and the creases fold freely without any resistance. In real materials, the creases can offer some resistance to folding as well. We can model such complications by introducing a torsional spring on each crease. The energy model will be modified to

$$
\begin{aligned}
E & =E_{\text {Vertex }}+E_{\text {Face }}+E_{\text {Crease }} \\
& =\sum_{a} T_{a}^{2}+\frac{1}{2} \kappa_{f} \sum_{\text {faces }} \rho_{f}^{2}+\frac{1}{2} \kappa_{c} \sum_{\text {creases }} \rho^{2} .
\end{aligned}
$$

We compared the branching statistics of a $3 \times 3$ looped disordered mesh [Fig. 10(e), blue] by considering $5 \times 10^{3}$ initial random torque schemes in the simplified and stiff crease models. Compared to the supplied external torque, the stiffness of faces was assigned a value $k_{f}=0.1 \tau$, and the stiffness of creases was $k_{c}=0.02 \tau$. The numbers of 
Branch \#1

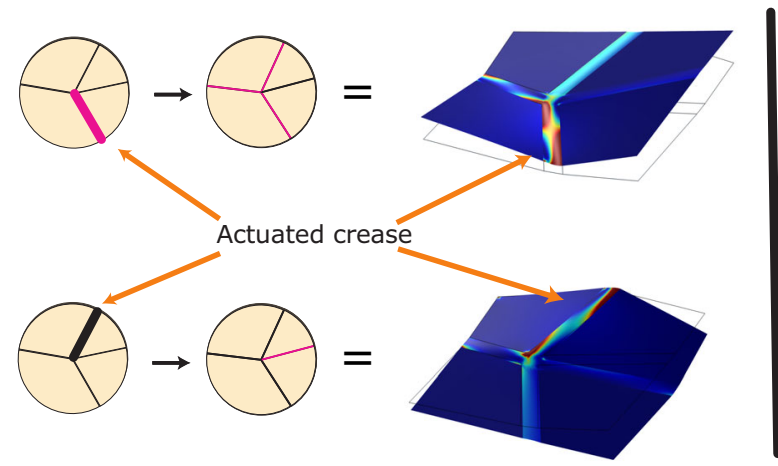

Branch \#2

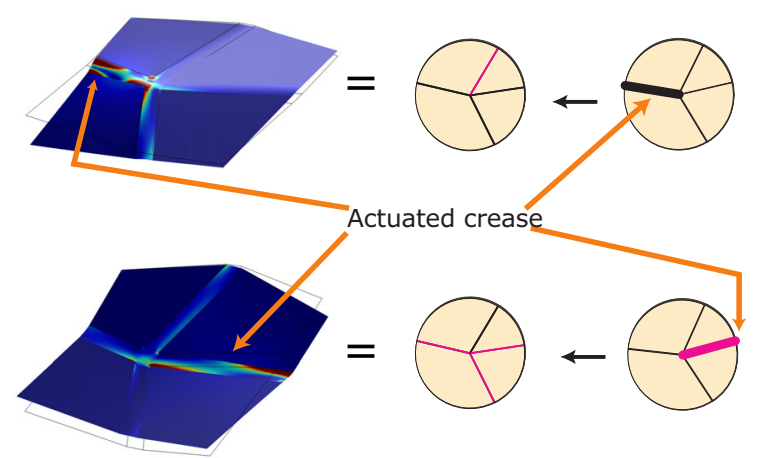

FIG. 9. Finite-element models of realistic sheets, simulated with COMsOL Multiphysics, show that the mechanical advantage rule identifies the right folding branch. When a torque is applied to just one crease, the resulting branch is predicted by the mechanical advantage rule [Fig. 2(c)]. We used the COMSOL shell model to simulate folding a realistic origami vertex (experiencing elastic strains). The folded configurations match those expected from our simplified vertex model defined only by vertex constraints, even though the COMSOL model accounts for many real-world complications not accounted for by our simple model (e.g., finite crease stiffness and thickness, delocalized bending and stretching). The pattern parameters are length approximately $0.2 \mathrm{~m}$, crease width $0.015 \mathrm{~m}$, thickness $10^{-4} \mathrm{~m}$. Material parameters of the faces are density $\rho=1760 \mathrm{~kg} / \mathrm{m}^{3}$, Young's modulus $Y=8 \times 10^{8}$ Pa. Material parameters of the creases are density $\rho=930 \mathrm{~kg} / \mathrm{m}^{3}$, Young's modulus $Y=5 \times 10^{6} \mathrm{~Pa}$.

branches found for these two models were different by less than 1\%. As shown in Figs. 10(b) and 10(c) the statistics of the landscape are also very similar; the energy of minima and their attractor region sizes have nearly identical cumulative distribution functions, showing that the glassy attractor structure is essentially the same. Another metric by which we can estimate how similar the landscapes are is by pairing up the closest attractors (in the dot product sense) between the two landscapes. We find that the majority (approximately 64\%) of the folding torque schemes lead to paired up branches in the two models. In contrast, only rarely does the same torque lead to approximately orthogonal branches in both models, showing that the expected resulting branches are well correlated.

\section{Mechanical advantage rule}

In the idealized model considered in the paper, we find that the mechanical advantage rule predicts the branching of a 4-vertex for any applied torque. The simplified 4vertex will always fold into the branch that has the larger dot product with the applied torque. A graphical representation of this fact is seen in Fig. 2(c), in which the ridge in the angular energy function is always located halfway between the two minima.

This idealization will be modified for more complicated (and realistic) elastic models, for which the energy ridge might be shifted closer to one of the branching minima. One way to model such alterations is again considering stiff creases (modified with torsional springs). We find that the energy ridge can be moved towards one of the minima by increasing the stiffness of creases [Fig. 10(a)]. However, a significant deviation requires very stiff creases that overwhelm the vertex constraints (meaning the origami pattern will more effectively bend and stretch than fold). As long as the pattern retains its folding topology, when creases are not too stiff and can be practically folded, the mechanical advantage rule is approximately correct.

To test this prediction under more real-world complications, we simulated an origami vertex made of realistic materials with COMSOL Multiphysics (Fig. 9). This COMSOL model includes finite thickness of the sheet, creases of finite width, thickness and stiffness, face bending and stretching that is distributed generally over the sheet, among other complications that were not accounted for in our simplified model. Despite this, the mechanical advantage rule does in fact predict the correct branch folded by the tested initial torque schemes.

Finally, we emphasize that real-world deviations from the mechanical advantage rule can only change the relative attractor size of the two branches at each vertex. The qualitative glassy landscape relies only on the existence of two branches at each vertex and is not tied to their precise size.

\section{Manufacturing errors}

An additional complication to realistic origami patterns is the impossibility of perfectly manufacturing a designed pattern. Any physical manufacturing process will inevitably introduce errors in the pattern by placing vertices slightly off of their designed position. The effect of such errors on the energy of the designed branch was discussed previously [39], but even if the pattern could still fold into the designed branch, it remains possible that the designed actuation scheme will not work. This might happen if the designed applied torques (e.g., chosen by considering the folding islands method) now lead to a different uncorrelated high-energy minimum (distractor) in the landscape. 


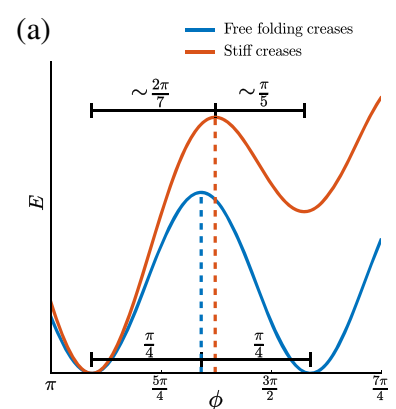

(e)

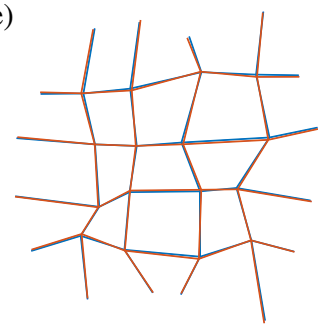

(b)

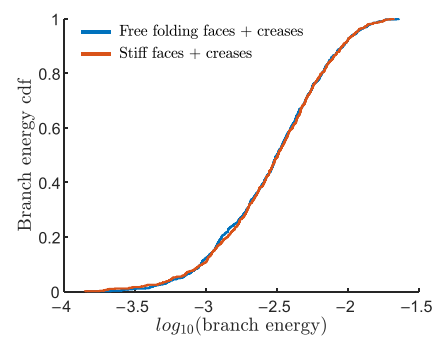

(f)

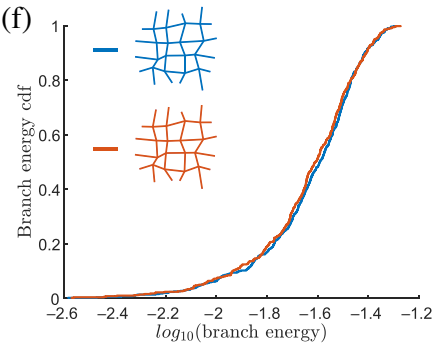

(c)

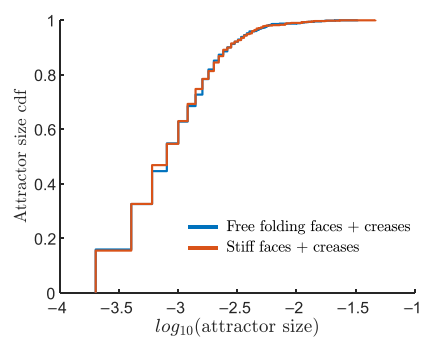

(g)

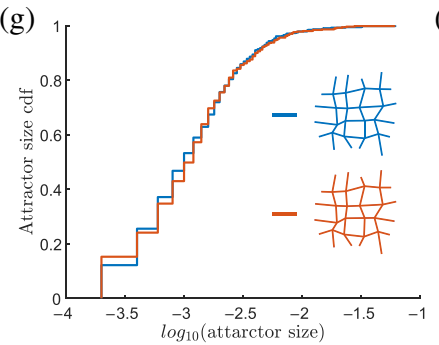

(d)
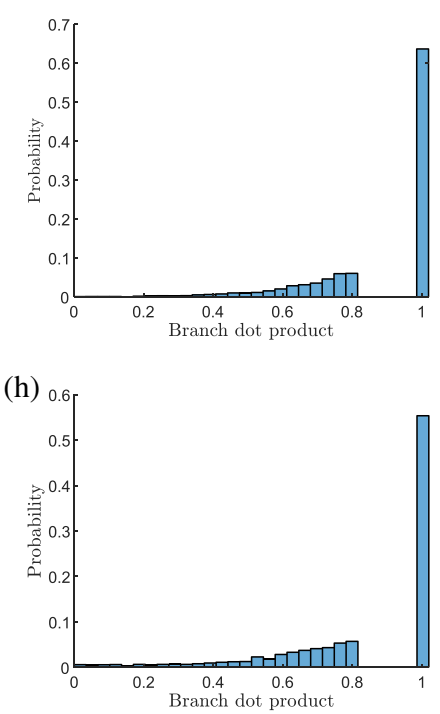

FIG. 10. Varying material models changes landscape details but maintains underlying structure of exponential minima. (a) Simulating a 4-vertex with stiff creases results in the same two modes of an idealized vertex, as long as the creases are not overwhelmingly stiff. The shift in minima position causes a moderate deviation from the mechanical advantage rule for sufficiently stiff creases. (b) and (c) Comparing a pattern with free-folding faces and creases to the same pattern with stiff faces and creases, the number of branches remains similar. Furthermore, the distribution of branch energies and attractor sizes is statistically indistinguishable, implying that the glassy structure retains the same statistics. (d) When branches of the patterns with free-folding and stiff faces or creases are paired up, we find that most random attempts to fold the system enter the same branch for both patterns, showing how corresponding branches remain "close" in configuration space. (e) Realistic origami structures are expected to have manufacturing errors in vertex placement. The blue and orange pattern's vertices differ in location by $2 \%$ of the mean lattice spacing. (f)-(h) The same metrics as in (b)-(d) show how the landscape of patterns with manufacturing errors retain the same statistics as the originals.

We simulated two patterns, one with vertices displaced by $2 \%$ compared to the other [Fig. 10(e)]. To check whether the energy landscape changed considerably as a result of this perturbation, we computed the same metrics as discussed previously in this section [Figs. 10(f) and 10(g)] for $5 \times 10^{3}$ random torque schemes. The statistics of the energy landscape are once more essentially the same, with the same distribution of branch energies and attractor sizes. Moreover, paired-up branches between the two patterns (by dot product proximity) have highly overlapping attractors, as approximately $55 \%$ of the random folding torques fold into paired-up branches [Fig. 10(h)]. However, there is indeed a significant probability that a designed actuation scheme for the original pattern will fail for the displaced pattern (in this sample, approximately 45\%). Still, by controlling the manufacturing error, one could make the energy landscape of the displaced pattern a better approximation of the original landscape, such that the success of actuation would be nearly guaranteed.

\section{APPENDIX D: DOT PRODUCT AND ATTRACTOR SIZE}

In the main paper, we mostly considered actuators applying equal torques to reflect many applications where the precise locations of actuators are easily controlled but the precise magnitudes of applied torque are not.
Here, we study vectors of folding torques $\vec{\tau}$ whose components might be variable in magnitude across the pattern. We might expect folding to be successful if the dot product $D=\vec{\tau} \cdot \vec{\rho}_{\text {desired }} /\|\vec{\tau}\|\left\|\vec{\rho}_{\text {desired }}\right\|$ between the applied torque and the desired mode is higher than the dot products $\vec{\tau} \cdot \vec{\rho}_{\alpha} /\|\vec{\tau}\|\left\|\vec{\rho}_{\alpha}\right\|$ with all distractor modes $\alpha$. In practice, how large does $D$ need to be for successful folding of the desired branch?

To determine the dot product needed, we actuated folding using random torques as in the main paper but now characterized success of folding as a function of the dot product (Fig. 11). We see that, for sufficiently large patterns, a dot product of $D \sim 1$ between the applied force and the desired branch is needed to have a significant chance of success.

Naively, a high dot product might seem easy to achieve; but note that in high dimensions (e.g., $4 \times 4$ patterns have a $60 d$ configuration space), a vanishingly small fraction of all vectors (e.g., $\vec{\tau}$ ) has a non-negligible dot product $D$ with any fixed vector (e.g., $\vec{\rho}_{\text {desired }}$ ).

In conclusion, large patterns require a high dot product $D \sim \vec{\tau} \cdot \vec{\rho}_{\text {desired }}$ between the applied vector of torques $\vec{\tau}$ and the folding angles of the desired branch $\vec{\rho}_{\text {desired }}$ to fold successfully; since random vectors have vanishing dot products in high-dimensional spaces, high dot product $D$ is increasingly difficult to achieve. Such finely tuned 


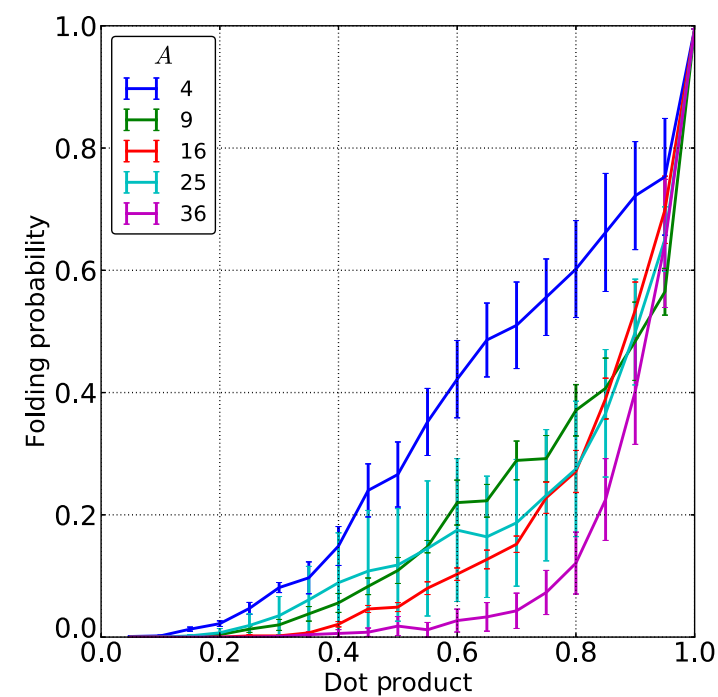

FIG. 11. Large meshes require the applied vector of torques $\vec{\tau}$ to be closely aligned with the folding angles $\vec{\rho}_{\text {desired }}$ of the desired branch for successful folding. By folding random quadrilateral meshes of different sizes with random $\vec{\tau}$ and determining the success of folding, we find that the dot product $D=$ $\vec{\tau} \cdot \vec{\rho}_{\text {desired }} /\|\vec{\tau}\|\left\|\vec{\rho}_{\text {desired }}\right\|$ in a large quadrilateral mesh must be close to 1 . Since most vectors in high-dimensional space are orthogonal, only a vanishingly small fraction of applied vectors of torques can successfully fold the desired branch.

torques applied to every crease of a large pattern defeat the purpose of building self-folding origami structures.

\section{APPENDIX E: COMPUTATION OF FOLDING ISLANDS}

As discussed in the main text, identifying the folding islands of the pattern provides a design principle for actuation schemes that successfully fold the desired branch. We find that choosing actuators whose union of folding islands covers the entire pattern dramatically improves the probability of successful folding. In this section, we outline how folding islands are found and list some limitations regarding these procedures.

The folding island of a crease is defined as the largest contiguous region that can be folded successfully when cut out of the pattern. In principle, there might exist distinct folding islands of equal area for a given actuator; in this case, we associate both of these maximal folding islands to the actuator with the understanding that when combined with another actuator, the more favorable folding island can be used. However, we did not find any such instances in our numerical exploration of patterns.

The method we employ to compute the folding island of a given crease is directly derived from the definition. (a) For the given pattern [e.g., Fig. 5(a)] and a given actuator crease, we first try folding a subpattern composed of just the two vertices connected to it. (b) We check whether any vertex in this set folds into the desired branch. If so, such vertices are included into the folding island. (c) We then enumerate the candidate vertices for the folding island found at the boundary of the current folding island-i.e., we enumerate all vertices connected to vertices already in the folding island. We pick a random member from this list, add it to the folding island, and attempt to fold. (d) If the putative extended folding island folds correctly (including the new added vertex), the new vertex is included into the folding island. (e) We go back to step (c) and repeat until we can no longer add any vertices that fold successfully when the initial crease is actuated. When the process terminates, we are guaranteed a correctly folding region that is not contained in any larger folding region. (f) We repeat the entire process multiple times from scratch to explore alternative orderings of growth. The largest resulting folding island over many runs is taken to be the true folding island.

Given enough trials, the algorithm will pick out the largest folding subset (defined to be the true folding island). With finite running time, the algorithm can, in principle, underestimate the size of the true largest folding island. In such a case, the heuristic of UFIs is an even better metric than that implied by Fig. 5, since some actuation schemes that worked at UFI $<1$ should actually be described by $\mathrm{UFI}=1$.

In practice, we found that testing putative boundary vertices by order of their distance (instead of a random order) from the actuator crease was particularly effectivethis method quickly provided folding islands at least as large as those obtained after a small fixed number of random order trials, but it is much faster. We used this faster method to compute the large number of folding islands needed for Fig. 5.

\section{Design principle for actuator placement}

Our results suggest a design principle for actuator placement: We first work out the folding island for each crease (through simulation or experiments). Then, using algorithms for the set covering problem [53], we can identify minimal combinations of actuator creases whose folding islands cover the whole pattern. (The set covering problem is a classic problem in computer science; given a set of subsets $S$ of a "universe" set $U$, one is asked to find a minimal combination of subsets $S$ whose union is $U$. Many algorithms exist [53]. Here, $U$ represents our vertices and $S$ represents the set of folding islands of each vertex in the pattern.) Thus we have replaced a mechanically difficult problem at the time of actuation with a computationally difficult problem at the time of design. Further, by reducing properties of actuator combinations to a property of single actuators (i.e., their folding island), the above proposal is vastly more feasible than an exhaustive computational or experimental search through all combinations of actuators.

We can offer a rule of thumb for identifying which creases are likely to have large folding islands. Each vertex 
may be considered to "point" to two of its neighbors, along the transverse line whose two creases have the same sign of folding in the desired state. If the folding propagates from an actuator to the vertex via one of these neighbors, the vertex will fold correctly, by the mechanical advantage rule discussed above. Paths of pointing can be constructed, and if a crease is pointed to by many, long paths, it will tend to have a large folding island. The effect of loops and material complications means that this rule gives only a guide, not an exact prescription.

Applying these ideas to common patterns, note that Miura-Ori has a sequence of long narrow folding islands along the zigzag creases that fold in the same MV state. We previously showed [39] that origami patterns can be classified into three classes: natural, seminatural, and unnatural. Natural patterns are the easiest to design and resemble Miura-Ori in that they contain one direction along which rows of creases are either all mountains or valleys; columns of creases in the perpendicular direction have alternating MV states. Such patterns should generally be expected to have long thin islands along the homogeneous direction. In contrast, seminatural and unnatural patterns [e.g., that in Fig. 5(j)] tend to have compact islands.

[1] E. A. Peraza-Hernandez, D. J. Hartl, R. J. Malak, Jr., and D. C. Lagoudas, Origami-Inspired Active Structures: A Synthesis and Review, Smart Mater. Struct. 23, 094001 (2014).

[2] J. L. Silverberg, A. A. Evans, L. McLeod, R. C. Hayward, T. Hull, C. D. Santangelo, and I. Cohen, Using Origami Design Principles to Fold Reprogrammable Mechanical Metamaterials, Science 345, 647 (2014).

[3] S. Pellegrino, Deployable Structures (Springer, New York, 2014).

[4] P. M. Reis, H. M. Jaeger, and M. van Hecke, Designer Matter: A Perspective, Extreme Mech. Lett. 5, 25 (2015).

[5] R. J. Lang, The Science of Origami, Phys. World 20, 30 (2007).

[6] E. D. Demaine and J. O'Rourke, Geometric Folding Algorithms: Linkages, Origami, Polyhedra (Cambridge University Press, Cambridge, England, 2007).

[7] T. Tachi, Evolution and Trends in Design, Analysis and Construction of Shell and Spatial Structures, in Proceedings of the International Association for Shell and Spatial Structures (IASS) Symposium 2009, Valencia, edited by A. Domingo and C. Lazaro (Universidad Politecnica de Valencia, Spain, 2009).

[8] T. Tachi, Geometric considerations for the design of rigid origami structures, in Proceedings of the International Association for Shell and Spatial Structures (IASS) Symposium 2010, Shanghai (2010).

[9] J.-H. Na, A. A. Evans, J. Bae, M. C. Chiappelli, C. D. Santangelo, R. J. Lang, T. C. Hull, and R. C. Hayward, Programming Reversibly Self-Folding Origami with Micropatterned Photo-Crosslinkable Polymer Trilayers, Adv. Mater. 27, 79 (2015).
[10] L. H. Dudte, E. Vouga, T. Tachi, and L. Mahadevan, Programming Curvature Using Origami Tessellations, Nat. Mater. 15, 583 (2016).

[11] S. Hormoz and M. P. Brenner, Design Principles for SelfAssembly with Short-Range Interactions, Proc. Natl. Acad. Sci. U.S.A. 108, 5193 (2011).

[12] V. S. Pande, A. Y. Grosberg, and T. Tanaka, Heteropolymer Freezing and Design: Towards Physical Models of Protein Folding, Rev. Mod. Phys. 72, 259 (2000).

[13] W. M. Jacobs, A. Reinhardt, and D. Frenkel, Communication: Theoretical Prediction of Free-Energy Landscapes for Complex Self-Assembly., J. Chem. Phys. 142, 021101 (2015).

[14] Z. Zeravcic, V. N. Manoharan, and M. P. Brenner, Size Limits of Self-Assembled Colloidal Structures made using Specific Interactions, Proc. Natl. Acad. Sci. U.S.A. 111, 15918 (2014).

[15] A. Murugan, J. Zou, and M. P. Brenner, Undesired Usage and the Robust Self-Assembly of Heterogeneous Structures, Nat. Commun. 6, 6203 (2015).

[16] V. I. Abkevich, A. M. Gutin, and E. I. Shakhnovich, Free Energy Landscape for Protein Folding Kinetics: Intermediates, Traps, and Multiple Pathways in Theory and Lattice Model Simulations, J. Chem. Phys. 101, 6052 (1994).

[17] M. Karplus, The Levinthal Paradox: Yesterday and Today, Folding Des. 2, S69 (1997).

[18] C. Levinthal, Mossbauer Spectroscopy in Biological Systems, Proceedings of a Meeting held at Allerton House, Monticello, 1969 (University of Illinois Press, Champaign, 1969), pp. 22-24.

[19] P. Crescenzi, D. Goldman, C. Papadimitriou, A. Piccolboni, and M. Yannakakis, On the Complexity of Protein Folding, J. Comput. Biol. 5, 423 (1998).

[20] J. T. Ngo, J. Marks, and M. Karplus, Computational Complexity, Protein Structure Prediction, and the Levinthal Paradox, in The Protein Folding Problem and Tertiary Structure Prediction, edited by K. M. Merz, Jr., and S. M. Le Grand (Birkhäuser, Boston, 1994), pp. 433-506.

[21] G. Biroli, S. Cocco, and R. Monasson, Phase Transitions and Complexity in Computer Science: An Overview of the Statistical Physics Approach to the Random Satisfiability Problem, Physica (Amsterdam) 306A, 381 (2002).

[22] M. Bern and B. Hayes, The Complexity of Flat Origami, Proceedings of the Seventh Annual ACM-SIAM Symposium on Discrete Algorithms (1996), pp. 175-183.

[23] Z. Abel, J. Cantarella, E. D. Demaine, D. Eppstein, T. C. Hull, J.S. Ku, R. J. Lang, and T. Tachi, Rigid Origami Vertices: Conditions and Forcing Sets, J. Comput. Geom. 7, 171 (2016).

[24] B. Ballinger, M. Damian, D. Eppstein, R. Flatland, J. Ginepro, and T. Hull, Minimum Forcing Sets for Miura Folding Patterns, in Proceedings of the Twenty-Sixth Annual ACM-SIAM Symposium on Discrete Algorithms, SODA '15, Philadelphia, PA, USA, 2015, pp. 136-147. Society for Industrial and Applied Mathematics.

[25] E. M. Arkin, M. A. Bender, E. D. Demaine, M. L. Demaine, J. S. B. Mitchell, S. Sethia, and S. S. Skiena, When Can You Fold a Map? Comput. Geom. 29, 23 (2004).

[26] S. Pandey, M. Ewing, A. Kunas, N. Nguyen, D. H. Gracias, and G. Menon, Algorithmic Design of Self-Folding Polyhedra, Proc. Natl. Acad. Sci. U.S.A. 108, 19885 (2011). 
[27] E. Hawkes, B. An, N. M. Benbernou, H. Tanaka, S. Kim, E. D. Demaine, D. Rus, and R. J. Wood, Programmable Matter by Folding, Proc. Natl. Acad. Sci. U.S.A. 107, 12441 (2010).

[28] B. An, N. Benbernou, E. D. Demaine, and D. Rus, Planning to Fold Multiple Objects from a Single Self-Folding Sheet, Robotica 29, 87 (2011).

[29] T. Tachi and T. C. Hull, Self-Foldability of Rigid Origami, in ASME 2016 International Design Engineering Technical Conferences and Computers and Information in Engineering Conference American Society of Mechanical Engineers, 2016, pp. V05BT07A029-V05BT07A029.

[30] D. A. Huffman, Curvature and Creases: A Primer on Paper, IEEE Trans. Comput. C-25, 1010 (1976).

[31] S. Waitukaitis, R. Menaut, B. Gin-Ge Chen, and M. van Hecke, Origami Multistability: From Single Vertices to Metasheets, Phys. Rev. Lett. 114, 055503 (2015).

[32] T. Tachi, Geometric Considerations for the Design of Rigid Origami Structures, in Proceedings of the International Association for Shell and Spatial Structures (IASS) Symposium, Vol. 12, 2010, pp. 458-460.

[33] S.-M. Belcastro and T. C. Hull, Modelling the Folding of Paper into Three Dimensions Using Affine Transformations, Linear Algebra Appl. 348, 273 (2002).

[34] T. Kawasaki, On the Relation between Mountain-Creases and Valley-Creases of a Flat Origami, in Proceedings of the 1st International Meeting of Origami Science and Technology, 1989, pp. 229-237.

[35] T. Hull, On the Mathematics of Flat Origamis, Congr. Numer. 100, 215 (1994).

[36] J. L. Silverberg, J.-H. Na, A. A. Evans, B. Liu, T. C. Hull, C. D. Santangelo, R. J. Lang, R. C. Hayward, and I. Cohen, Origami Structures with a Critical Transition to Bistability Arising from Hidden Degrees of Freedom, Nat. Mater. 14, 389 (2015).

[37] K. Binder and A. P. Young, Spin Glasses: Experimental Facts, Theoretical Concepts, and Open Questions, Rev. Mod. Phys. 58, 801 (1986).

[38] T. Tachi, Design of Infinitesimally and Finitely Flexible Origami Based on Reciprocal Figures, J. Geom. Graph. 16, 223 (2012).

[39] M. B. Pinson, M. Stern, A. C. Ferrero, T. A. Witten, E. Chen, and A. Murugan, Self-Folding Origami at Any Energy Scale, Nat. Commun. 8, 15477 (2017).
[40] A. Lobkovsky, S. Gentges, H. Li, D. Morse, and T. A. Witten, Scaling Properties of Stretching Ridges in a Crumpled Elastic Sheet, Science 270, 1482 (1995).

[41] T. A. Witten, Stress Focusing in Elastic Sheets, Rev. Mod. Phys. 79, 643 (2007).

[42] B. Gin-ge Chen and C. D. Santangelo, Branches of Triangulated Origami near the Flat State, arXiv:1706.03869.

[43] Y. V. Fyodorov, Complexity of Random Energy Landscapes, Glass Transition, and Absolute Value of the Spectral Determinant of Random Matrices, Phys. Rev. Lett. 92, 240601 (2004).

[44] B. Berger and T. Leighton, Protein Folding in the Hydrophobic-Hydrophilic (HP) Model Is NP-Complete, J. Comput. Biol. 5, 27 (1998).

[45] F. Krzakala and L. Zdeborova, Phase Transitions and Computational Difficulty in Random Constraint Satisfaction Problems, J. Phys. Conf. Ser. 95, 012012 (2008).

[46] I. Lynce and O. Joël, Sudoku as a SAT Problem, in Proceedings of the Ninth International Symposium on Artificial Intelligence and Mathematics, Aimath 2006, Fort Lauderdale (Springer, New York, 2006.

[47] J. M. Zanardi Ocampo, P. O. Vaccaro, T. Fleischmann, T.-S. Wang, K. Kubota, T. Aida, T. Ohnishi, A. Sugimura, R. Izumoto, M. Hosoda, and S. Nashima, Optical Actuation of Micromirrors Fabricated by the Micro-Origami Technique, Appl. Phys. Lett. 83, 3647 (2003).

[48] K. Kuribayashi, K. Tsuchiya, Z. You, D. Tomus, M. Umemoto, T. Ito, and M. Sasaki, Self-Deployable Origami Stent Grafts as a Biomedical Application of Ni-Rich TiNi Shape Memory Alloy Foil, Mater. Sci. Eng. 419, 131 (2006).

[49] T. S. Shim, S.-H. Kim, C.-J. Heo, H. C. Jeon, and S.-M. Yang, Controlled Origami Folding of Hydrogel Bilayers with Sustained Reversibility for Robust Microcarriers, Angew. Chem., Int. Ed. Engl. 51, 1420 (2012).

[50] S. M. Felton, M. T. Tolley, B. Shin, C. D. Onal, E. D. Demaine, D. Rus, and R. J. Wood, Self-Folding with Shape Memory Composites, Soft Matter 9, 7688 (2013).

[51] O. Gottesman, E. Efrati, and S. M. Rubinstein, Furrows in the Wake of Propagating d-Cones, Nat. Commun. 6, 7232 (2015).

[52] A. J. Wood, Witten's Lectures on Crumpling, Physica (Amsterdam) 313A, 83 (2002).

[53] V. V. Vazirani, Approximation Algorithms (Springer Science \& Business Media, New York, 2013). 